\title{
Exercise cardiovascular magnetic resonance: feasibility and development of biventricular function and great vessel flow assessment, during continuous exercise accelerated by Compressed SENSE: preliminary results in healthy volunteers
}

\author{
Thomas P. Craven ${ }^{1}$ (D) Nicholas Jex ${ }^{1} \cdot$ Pei G. Chew ${ }^{1} \cdot$ David M. Higgins $^{2} \cdot$ Malenka M. Bissell $^{1} \cdot$ Louise A. E. Brown $^{1}$. \\ Christopher E. D. Saunderson ${ }^{1} \cdot$ Arka Das $^{1}$ - Amrit Chowdhary ${ }^{1}$ - Erica Dall'Armellina ${ }^{1}$ - Eylem Levelt ${ }^{1}$. \\ Peter P. Swoboda ${ }^{1}$. Sven Plein ${ }^{1}$. John P. Greenwood ${ }^{1}$
}

Received: 29 July 2020 / Accepted: 25 September 2020 / Published online: 4 October 2020

(c) The Author(s) 2020

\begin{abstract}
Purpose Exercise cardiovascular magnetic resonance (Ex-CMR) typically requires complex post-processing or transient exercise cessation, decreasing clinical utility. We aimed to demonstrate the feasibility of assessing biventricular volumes and great vessel flow during continuous in-scanner Ex-CMR, using vendor provided Compressed SENSE (C-SENSE) sequences and commercial analysis software (Cvi42).

Methods 12 healthy volunteers (8-male, age: $35 \pm 9$ years) underwent continuous supine cycle ergometer (Lode-BV) ExCMR (1.5T Philips, Ingenia). Free-breathing, respiratory navigated C-SENSE short-axis cines and aortic/pulmonary phase contrast magnetic resonance (PCMR) sequences were validated against clinical sequences at rest and used during low and moderate intensity Ex-CMR. Optimal PCMR C-SENSE acceleration, C-SENSE-3 (CS3) vs C-SENSE-6 (CS6), was further investigated by image quality scoring. Intra-and inter-operator reproducibility of biventricular and flow indices was performed.

Results All CS3 PCMR image quality scores were superior $(\mathrm{p}<0.05)$ to CS6 sequences, except pulmonary PCMR at moderate exercise. Resting stroke volumes from clinical PCMR sequences correlated stronger with CS3 than CS6 sequences. Resting biventricular volumes from CS3 and clinical sequences correlated very strongly ( $r>0.93)$. During Ex-CMR, biventricular end-diastolic volumes (EDV) remained unchanged, except right-ventricular EDV decreasing at moderate exercise. Biventricular ejection-fractions increased at each stage. Exercise biventricular cine and PCMR stroke volumes correlated very strongly $(r \geq 0.9)$, demonstrating internal validity. Intra-observer reproducibility was excellent, co-efficient of variance $(\mathrm{COV})<10 \%$. Inter-observer reproducibility was excellent, except for resting right-ventricular, and exercise bi-ventricular end-systolic volumes which were good (COV 10-20\%).

Conclusion Biventricular function, aortic and pulmonary flow assessment during continuous Ex-CMR using CS3 sequences is feasible, reproducible and analysable using commercially available software.
\end{abstract}

Keywords Exercise cardiovascular magnetic resonance $\cdot$ Exercise $\cdot$ Flow $\cdot$ Ventricular function

\begin{tabular}{|c|c|c|c|}
\hline & & Abbrevia & \\
\hline & & BPM & Beats per minute \\
\hline & & CAD & Coronary artery disease \\
\hline & & CMR & Cardiovascular magnetic resonance \\
\hline 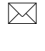 & Thomas P. Craven & CPET & Cardiopulmonary exercise testing \\
\hline & thomascraven@ doctors.org.uK & CS3/CS6 & Compressed SENSE encoding accelerated \\
\hline 1 & Multidisciplinary Cardiovascular Research Centre \& & & by a factor of $3 / 6$ \\
\hline & The Division of Biomedical Imaging, Leeds Institute & $\mathrm{ECG}$ & Electrocardiogram \\
\hline & of Cardiovascular and Metabolic Medicine, University & EDV & End-diastolic volume \\
\hline & of Leeds, Leeds LS2 9J1, UK & EF & Ejection fraction \\
\hline
\end{tabular}




$\begin{array}{ll}\text { ESV } & \text { End systolic volume } \\ \text { Ex-CMR } & \begin{array}{l}\text { Exercise cardiovascular magnetic } \\ \text { resonance }\end{array} \\ \text { HR } & \text { Heart rate } \\ \text { HR }_{\max } & \text { Maximal heart rate } \\ \text { HRR } & \text { Heart rate reserve } \\ \mathrm{i} & \text { Indexed to body surface area } \\ \text { LV } & \text { Left ventricle } \\ \text { LVEF } & \text { Left ventricular ejection fraction } \\ \text { LVOT } & \text { Left ventricular outflow tract } \\ \text { LVSV } & \text { Left Ventricular Stroke volume } \\ \text { MPS-SPECT } & \text { Myocardial perfusion scintigraphy } \\ & \text { by single photon emission computed } \\ & \text { tomography } \\ \text { MR } & \text { Mitral regurgitation } \\ \text { MR-CPET } & \text { Magnetic resonance cardiopulmonary } \\ & \text { exercise testing } \\ \text { PCMR } & \text { Phase contrast magnetic resonance } \\ \text { RVEF } & \text { Right ventricular ejection fraction } \\ \text { RVOT } & \text { Right ventricular outflow tract } \\ \text { RVSV } & \text { Right ventricular stroke volume } \\ \text { SENSE } & \text { Sensitivity encoding } \\ \text { SV } & \text { Stroke volume } \\ \text { THR } & \text { Target heart rate } \\ & \end{array}$

\section{Introduction}

Stress cardiac imaging is an important tool in assessing valvular [1] and congenital heart disease [2] and has significantly improved the diagnostic accuracy for coronary artery disease (CAD) detection compared to exercise ECG [3, 4]. Cardiovascular magnetic resonance (CMR) has well established benefits over alternative imaging modalities and as such is the reference standard for bi-ventricular volume and functional assessment [5]. Pharmacological stress CMR is well established clinically, demonstrating superiority over myocardial perfusion scintigraphy by single photon emission computed tomography (MPS-SPECT) in the diagnosis $[6,7]$ and prognostication of CAD [8]. However, physical exercise allows a more detailed assessment of symptoms, functional state and haemodynamic response and has fewer adverse events compared to pharmacological stress $[9,10]$. As such, current guidelines advise physical exercise as the preferred method for stress imaging when feasible [11, 12]. Exercise CMR (Ex-CMR) combines the superior image quality of CMR with the preferred method of stress by physiological exercise. Despite research development over the past 3 decades, Ex-CMR is not widely utilised clinically. Treadmill Ex-CMR has demonstrated clinical utility and superiority over MPS-SPECT, in the detection of ischaemia in CAD [13]. However, heart rate reductions during transfer to the MR-scanner limit its clinical utility beyond CAD assessment and make assessment at multiple exercise intensities logistically difficult. In-scanner Ex-CMR with a supine ergometer overcomes this issue, but CMR scanning during exercise results in increased physical movement, respiratory artefacts and ECG gating artefacts, all of which increase with increasing workload [14]. Originally, Ex-CMR studies, using retrospective cardiac gating, performed imaging during exercise cessation and breath holding to overcome these issues [15], unfortunately both are non-physiological and reduce clinical utility. Progression to real-time imaging allowed free breathing during Ex-CMR [16]. The continued need for cardiac gating resulted in detrimental artefacts at maximal exercise and real time Ex-CMR studies assessing flow report the acquisition of a significant volume of flow data $(<25,000$ images per patient), requiring the use of an online graphics processing unit reconstruction system and prolonged post processing/analysis time [17]. The development of ungated real-time cine imaging solved the ECG gating issues, allowing biventricular volume assessment during maximal exercise [14]. Recently, combining this technique with ungated flow acquisition resulted in the first study assessing bi-ventricular volumes and aortic and pulmonary flow during continuous exercise [18]. Unfortunately, the un-gated real-time technique requires specialist software (for post hoc cardiac and respiratory gating) and prolonged post processing and analysis time, thus decreasing clinical utility and widespread attainability. Compressed SENSE (C-SENSE) is a novel parallel imaging technique, robust to respiratory motion and allows fast image acquisition whilst maintaining high image quality [19]. To our knowledge [20], C-SENSE has not previously been utilised in Ex-CMR. The aims of this study are to demonstrate the feasibility of assessing biventricular volume and flow during continuous exercise using vendor provided C-SENSE sequences and commercially available standard analysis software.

\section{Materials and methods}

\section{Design}

Protocol development and feasibility testing was achieved by: (1) developing a free-breathing C-SENSE protocol and validating this against our institute's standard clinical imaging sequences at rest; (2) determining the optimal acceleration of C-SENSE for PCMR sequences, for use in Ex-CMR, by assessing resting and exercise image quality and comparing the derived stroke volumes against standard clinical imaging sequences at rest; (3) utilising the validated C-SENSE protocol during continuous low and moderate exercise intensities to determine if the acquired biventricular volumes and flow have internal validity in terms of consistency of ventricular stroke volumes when derived separately 
from cavity volumes and great vessel flow measurements, and whether they are concordant with expected supine exercise physiology.

This study was approved by a local ethics committee in England (Yorkshire and the Humber-Leeds East 18/ $\mathrm{YH} / 0168)$. All participants provided written informed consent. All Ex-CMR studies were performed at the Leeds General Infirmary, UK.

\section{Study population}

12 healthy volunteers ( 8 male, 4 female), aged $35 \pm 9$ years (mean \pm standard deviation) (range 23-56 years) underwent CMR at rest and during continuous exercise using the Lode BV supine bicycle ergometer. Participants were of a healthy weight (BMI 23.9 \pm 2.3 ) and of varying levels of physical fitness, performing regular exercise between 0.5 and $15 \mathrm{~h}$ a week (mean $5.0 \pm 3.5 \mathrm{~h}$ ). All healthy volunteers had no significant co-morbidities and no contraindications to exercise testing as per American heart association guidelines [21].

\section{Exercise protocol}

Participants performed supine cycle ergometer (Lode BV, Netherlands) (Fig. 1) exercise during CMR using heart rate reserve (HRR) and an age predictive maximal heart rate model [22], to prescribe individualised low (30-39\% HRR) and moderate (40-59\% HRR) exercise intensities. After completion of resting imaging, participants exercised with no resistance, 0 Watts (W), for $1 \mathrm{~min}$ at a cycling cadence of 60-70 rpm (with verbal feedback given to maintain this) then at an increase of $25 \mathrm{~W}$ every 2 min until 'low intensity' target heart rate (THR) was achieved; once THR was achieved smaller alterations in resistance wattage were made to maintain THR. HR was stabilised for $30 \mathrm{~s}$ prior to initiating imaging. After completion of imaging at low exercise intensity, resistance was increased by $25 \mathrm{~W}$ initially and every 2 min until the prescribed moderate intensity was reached and HR stabilised for $30 \mathrm{~s}$ prior to initiating imaging. Exercise performed was continuous and all exercise imaging acquired during free-breathing, with the use of straps around the patient and receiver coil to reduce exercise motion artefact (Fig. 1). Participants perceived rate of exertion were assessed on the Borg scale after exercise cessation, to ensure correlation with prescribed intensity [23].

\section{CMR imaging}

CMR imaging was performed on a dedicated cardiovascular 1.5T MR system (Philips Ingenia system, Best, Netherlands). Initial survey and cine imaging was performed including: vertical long axis, horizontal long axis, left ventricular outflow tract (LVOT) and right ventricular outflow tract
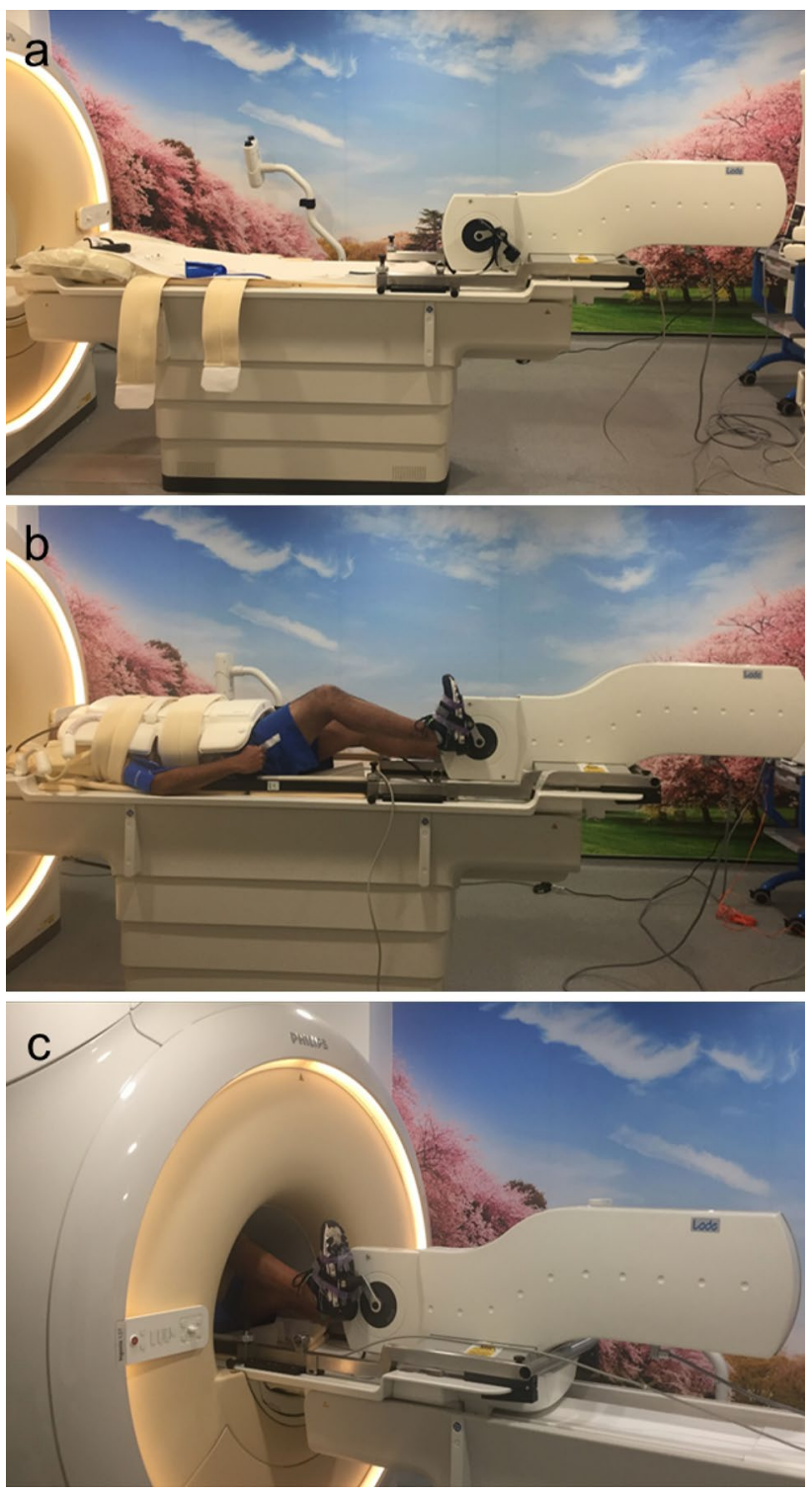

Fig. 1 The Lode BV supine cycle ergometer before (a), during set up (b) and during use (c)

(RVOT) views. At rest, our institute's standard clinical protocol to assess biventricular volumes, aortic and pulmonary flow was performed to validate the novel C-SENSE protocol against. The C-SENSE protocol was used at rest and during continuous exercise to low and moderate intensities.

\section{Standard clinical protocol}

Biventricular function was assessed using a breath-hold multi-phase, multi-slice short axis cine imaging stack (10 mm, no gap, 30 phases, SENSE 2). Great vessel flow was assessed from aortic and pulmonary through-plane phase contrast velocity mapping acquired during breathhold (SENSE 2) and a separate free-breathing acquisition 
(no parallel imaging) to ensure a comprehensive comparison with the novel C-SENSE protocol.

\section{C-SENSE protocol}

The evaluation protocol involved biventricular function assessment by free-breathing, respiratory navigated, continuous cine imaging in short axis geometry (10 mm, no gap) accelerated by a C-SENSE factor of 3 (CS3). Great vessel flow was assessed by aortic and pulmonary through-plane phase-contrast imaging, with two separate free-breathing acquisitions using C-SENSE 3 (CS3) and C-SENSE 6 (CS6) acceleration. CS3 and CS6 flow acquisitions were acquired to investigate if a higher acceleration would result in better image quality as a faster acquisition may be less prone to respiratory artefact. Additional CMR imaging parameters are described below.

During exercise, the above evaluation C-SENSE protocol was used with the addition of free-breathing LVOT/RVOT cine imaging being performed to assess for movement during exercise and re-plan the phase contrast imaging geometry if required.

\section{CMR imaging parameters}

All image acquisitions, including cine imaging and PCMR imaging, were retrospectively cardiac gated. The clinical short axis cine imaging parameters were as follows: typical FOV $360 \times 300 \mathrm{~mm}$, TR $3.1 \mathrm{msec}$, TE $1.56 \mathrm{msec}$, flip angle $60^{\circ}$, SENSE factor 2, multishot TFE factor 12, TFE acquisition duration $37.4 \mathrm{~ms}$, phase percentage $67 \%$, slice thickness $10 \mathrm{~mm}, 0 \mathrm{~mm}$ gap, 30 phases, in-plane spatial resolution acquired at $1.88 \times 1.88 \mathrm{~mm}$ and reconstructed to $1.25 \times 125 \mathrm{~mm}$, matrix $192 \times 158$, planned acquisition involved $7 \times 8$-s breath-holds. The C-SENSE short axis cine imaging parameters were as follows: typical FOV $300 \times 300 \mathrm{~mm}$, TR $2.4 \mathrm{msec}$, TE $1.21 \mathrm{msec}$, flip angle $60^{\circ}$, temporal resolution $32 \mathrm{msec}$. C-SENSE factor 3, multishot TFE factor 13, TFE acquisition duration $31.5 \mathrm{~ms}$, phase percentage $67 \%$, slice thickness $10 \mathrm{~mm}, 0 \mathrm{~mm}$ gap, in-plane spatial resolution acquired at $2.5 \times 2.5 \mathrm{~mm}$ and reconstructed to $1.34 \times 1.34 \mathrm{~mm}$, matrix $120 \times 120$, planned acquisition time 39 s. Respiratory navigation was used with the respiratory echo-based navigator positioned on the right hemi-diaphragm using a $5 \mathrm{~mm}$ acceptance window with continuous gating level drift.

Through-plane velocity encoded (VENC) PCMR was acquired at the aortic sino-tubular junction for aortic PCMR and in the main pulmonary artery (MPA) $1 \mathrm{~cm}$ superior to the valve for pulmonary PCMR. Resting VENC was set to $150 \mathrm{~cm} / \mathrm{s}$ and increased to $250 \mathrm{~cm} / \mathrm{s}$ during exercise; the VENC was increased further if aliasing occurred. To accommodate for potential through-plane motion during exercise, the CS3 and CS6 PCMR sequences were performed using a novel 'PCMR-imaging stack' acquiring $3 \times 8 \mathrm{~mm}$ overlapping PC-slices orthogonal to vessel flow (Fig. 2). Aortic PCMR sequences used a $-3 \mathrm{~mm}$ gap (thus the centre of the slices are spaced $5 \mathrm{~mm}$ apart) and the pulmonary flows had $-5 \mathrm{~mm}$ gap (thus the centre of the slices are spaced $3 \mathrm{~mm}$ apart). The increased overlap of the pulmonary PCMR sequences was to accommodate for the short length of the main pulmonary artery prior to bifurcation, which has led to difficulty performing pulmonary PCMR in previous ExCMR studies [24].

The clinical breath held (SENSE 2) and free-breathing CS3 and CS6 gradient echo PCMR sequences shared the following imaging parameters: typical FOV $350 \times 320 \mathrm{~mm}$, TR $4.9 \mathrm{msec}$, TE $2.9 \mathrm{msec}$, flip angle $15^{\circ}$, number of signal averages 1 , turbo field echo (TFE) factor 4 , slice thickness $8 \mathrm{~mm}$, 30 phases, phase percentage $67 \%$, acquired in-plane spatial resolution $2.5 \times 2.5 \mathrm{~mm}$ reconstructed to $1.22 \times 1.22 \mathrm{~mm}$, matrix $140 \times 128$, Cartesian sampling, planned acquisition time (per slice) of 13, 9 and 5 s for SENSE-2, CS3 and CS6 PCMR sequences respectively. The imaging parameters of the free-breathing standard clinical sequence (with no parallel imaging) were as follows: typical FOV $400 \times 280 \mathrm{~mm}$, TR $17 \mathrm{msec}$, TE $2.4 \mathrm{msec}$, flip angle $40^{\circ}$, number of signal averages 1, slice thickness $6 \mathrm{~mm}, 40$ phases, in-plane spatial

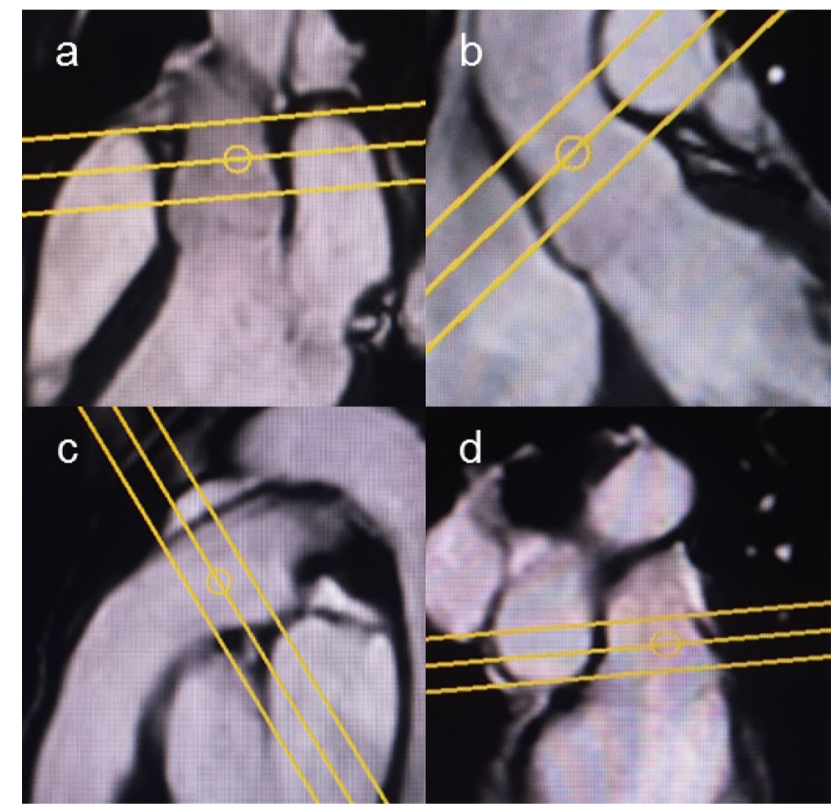

Fig. 2 Example of planning of aortic (a, b) and pulmonary (c, d) PCMR-stack. Aortic flow stack geometry, $8 \mathrm{~mm}$ slices with $-3 \mathrm{~mm}$ slice gap. Pulmonary PCMR-stack geometry, $8 \mathrm{~mm}$ slices with $-5 \mathrm{~mm}$ slice gap. a planning of aortic PCMR-stack in LVOT1 geometry. b planning of aortic PCMR-stack in LVOT2 geometry. c planning of pulmonary PCMR-stack in RVOT1 geometry. d planning of pulmonary PCMR-stack in RVOT2 geometry 
resolution $1.56 \times 2.23 \mathrm{~mm}$, matrix $256 \times 126$, Cartesian sampling, typical acquisition duration: $101 \mathrm{~s}$.

\section{CMR analysis}

Images were analysed using commercially available software (cvi42, Circle Cardiovascular Imaging, Calgary, AB, Canada). LV and RV endocardial contours were manually traced with the papillary muscles and trabeculations considered part of the ventricular blood pool and volumes calculated by summation of disks. Aortic and pulmonary flows were assessed by manually contouring the vessel in every phase. The CS3/CS6 PCMR-stack was assessed for the slice closest resembling the resting standard clinical acquisition to ensure all PCMR images had flow assessed at the same anatomical level. Image quality assessment was performed on all assessed PCMR images independently by two assessors (TC \& NJ), whom were blinded to each-others results. Images were graded on the following scale: 3 - excellent, 2- good, 1- adequate \& 0 - non diagnostic; the mean image quality scores from both assessors are presented.

\section{Statistical analysis}

Data were analysed using SPSS version 26 (IBM Corp.) and Microsoft Excel 2010. All continuous data were assessed for normality using Shapiro-Wilk test. Resting biventricular parameters comparing the breath-held standard clinical with CS3 respiratory navigated SA acquisitions were assessed by Pearsons correlation and the bias and limits of agreement by Bland-Altman plots. PCMR image quality scores were assessed by Wilcoxon signed ranks test and the stroke volume comparisons assessed by repeated measures ANOVA with Bonferroni post-test analysis. Repeated measures ANOVA with Bonferroni post-test analysis was used to compare cardiac volumetric and flow data between rest and different stages of exercise. Intra-observer analysis was performed by TC and inter-observer analysis by $\mathrm{NJ}$; reproducibility was assessed by the Coefficient of Variation (CV) test, the standard deviation of differences between observations divided by the mean and by intra-class correlation (ICC) with a two way random model for absolute agreement. $\mathrm{p}<0.05$ was considered statistically significant. Intra and inter-observer analysis was performed in a blinded method.

\section{Results}

13 healthy volunteers completed the study protocol, 1 volunteer was excluded due to ECG gating issues at moderate exercise intensity, leaving 12 healthy volunteers for analysis ( 8 male, age $35 \pm 9$ years, BMI $23.9 \pm 2.3 \mathrm{~kg} / \mathrm{m}^{2}$ ).

\section{Validation of free-breathing C-SENSE protocol at rest}

At rest, there were no significant differences between the biventricular volumes assessed by the standard clinical or novel CS3 short axis sequences, with all parameters demonstrating minimal bias and very strong correlation $(r>0.93$, $\mathrm{p} \leq 0.01$ ) (Table 1). Figure 3 demonstrates the typical image quality comparison between the resting breath-hold standard clinical and free-breathing CS3 short axis sequences. Mean resting aortic and pulmonary stroke volumes acquired from all 4 PCMR sequences were comparable, with CS3 and CS6 free-breathing flow showing minimal bias with both breathhold and free-breathing standard clinical flow sequences (Table 2). However, CS6 aortic flow measurements were more prone to underestimate aortic flow, with a bias of $-2.15 \mathrm{ml} / \mathrm{m}^{2} /$ cardiac cycle against the breath-hold clinical standard in comparison to a minimal bias of $-0.12 \mathrm{ml} /$ $\mathrm{m}^{2} /$ cardiac cycle using a CS3 flow sequence. Additionally, pulmonary stroke volumes from CS6 sequences only demonstrated moderate correlation with clinical free-breathing sequences $(r=0.655)$.

\section{Image quality scoring}

As expected, resting clinical breath-hold image quality scores for aortic and pulmonary flows were significantly higher compared to free-breathing sequences $(\mathrm{p}<0.01)$, except when compared with CS3 pulmonary flow $(\mathrm{p}=0.06)$ (Table 3). At rest, CS3 flow sequences had the highest image quality scores of all free-breathing sequences, including the free-breathing clinical sequence, and were significantly greater than CS6 sequences for aortic $(p=0.02)$ and pulmonary $(\mathrm{p}<0.01)$ flow. Figure 4 demonstrates the image quality of the different resting flow images acquired in the same patient. During exercise the image quality scores of CS3 aortic and pulmonary flow sequences were consistently higher than CS6 flow sequences. Indeed at moderate exercise intensity, five aortic and two pulmonary flow CS6 sequences were considered non-diagnostic, whereas all CS3 flow sequences were of adequate diagnostic quality. Due to the non-diagnostic image quality described in numerous CS6 flow acquisitions at moderate exercise intensity, the CS6 flow sequences were deemed unsuitable for Ex-CMR flow assessment and future studies.

\section{Supine bicycle exercise}

The participants' haemodynamic responses to supine bicycle exercise are displayed in Table 4. Participants' maintained within the target HR during each exercise stage increasing from $58 \pm 6 \mathrm{bpm}$ at rest, to $102 \pm 5 \mathrm{bpm}$ and $119 \pm 5 \mathrm{bpm}$ at low and moderate exercise respectively. Systolic BP rose 
Table 1 Validation of compressed SENSE 3 freebreathing sequences at rest vs breath-held clinical standard

\begin{tabular}{|c|c|c|c|c|c|c|c|c|}
\hline \multirow[t]{2}{*}{ Measurement } & \multicolumn{2}{|c|}{ Image sequence } & \multicolumn{4}{|c|}{ Bland altman } & \multicolumn{2}{|c|}{$\begin{array}{l}\text { Correlation coef } \\
\text { ficient }\end{array}$} \\
\hline & Clinical & CS3 & $\mathrm{RC}$ & Upper & Lower & Bias & $\mathrm{r}$ & p-value \\
\hline LVEDV (ml) & $165 \pm 39$ & $164 \pm 39$ & 7.05 & 6.34 & -7.76 & -0.71 & 0.996 & $<0.01$ \\
\hline LVEDVi $\left(\mathrm{ml} / \mathrm{m}^{2}\right)$ & $88.8 \pm 16$ & $88.5 \pm 16$ & 3.69 & 3.33 & -4.05 & -0.36 & 0.994 & $<0.01$ \\
\hline LVESV (ml) & $73 \pm 23$ & $71 \pm 23$ & 10.38 & 9.46 & -11.29 & -0.92 & 0.976 & $<0.01$ \\
\hline LVESVi (ml/m²) & $38.9 \pm 10$ & $38.4 \pm 11$ & 5.42 & 4.95 & -5.88 & -0.46 & 0.971 & $<0.01$ \\
\hline LVSV (ml) & $92 \pm 19$ & $93 \pm 19$ & 6.38 & 6.58 & -6.18 & 0.2 & 0.986 & $<0.01$ \\
\hline LVSVi (ml/m²) & $50 \pm 7$ & $50 \pm 7$ & 3.35 & 3.46 & -3.24 & 0.11 & 0.974 & $<0.01$ \\
\hline $\operatorname{LVEF}(\%)$ & $57 \pm 6$ & $57 \pm 6$ & 4.74 & 5.2 & -4.28 & 0.46 & 0.932 & $<0.01$ \\
\hline RVEDV (ml) & $166 \pm 36$ & $166 \pm 34$ & 8.59 & 9.21 & -7.96 & 0.62 & 0.995 & $<0.01$ \\
\hline RVEDVi (ml/m²) & $89.4 \pm 16$ & $89.8 \pm 15$ & 4.74 & 5.15 & -4.34 & 0.41 & 0.991 & $<0.01$ \\
\hline RVESV (ml) & $75 \pm 24$ & $75 \pm 21$ & 7.27 & 6.78 & -7.76 & -0.49 & 0.992 & $<0.01$ \\
\hline RVESVi (ml/m²) & $40.6 \pm 11$ & $40.4 \pm 10$ & 3.84 & 3.6 & -4.07 & -0.23 & 0.99 & $<0.01$ \\
\hline RVSV (ml) & $90 \pm 18$ & $91 \pm 17$ & 6.46 & 7.56 & -5.35 & 1.1 & 0.985 & $<0.01$ \\
\hline $\operatorname{RVSVi}\left(\mathrm{ml} / \mathrm{m}^{2}\right)$ & $48.8 \pm 8$ & $49.4 \pm 7$ & 3.51 & 4.15 & -2.88 & 0.63 & 0.977 & $<0.01$ \\
\hline RVEF (\%) & $55 \pm 7$ & $56 \pm 6$ & 2.82 & 3.23 & -2.42 & 0.4 & 0.985 & $<0.01$ \\
\hline Aortic SV (ml) & $89 \pm 18$ & $89 \pm 17$ & 7.75 & 7.44 & -8.05 & -0.31 & 0.978 & $<0.01$ \\
\hline Pulmonary SV (ml) & $90 \pm 15$ & $89 \pm 18$ & 12.42 & 11.21 & -13.63 & -1.21 & 0.944 & $<0.01$ \\
\hline
\end{tabular}

CS3 Compressed SENSE 3, EDV end-diastolic volume, EF ejection fraction, $E S V$ end-systolic volume, $H R$ heart rate, $i$ indexed to body surface area, $L V$ left ventricle, $R C$ repeatability coefficient, $R V$ right ventricle, $S V$ stroke volume
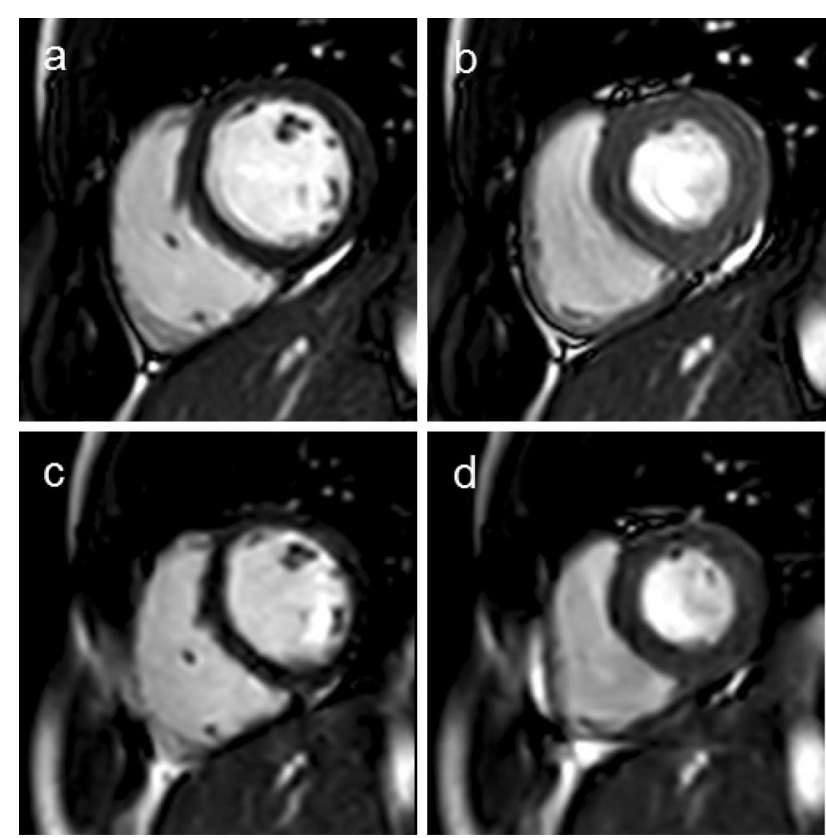

Fig. 3 Image quality comparison of resting short axis images acquired by clinical breath held SENSE 2 sequences at end-diastole (a) and end-systole (b) and Compressed SENSE 3 respiratory navigated sequences at and end-diastole (c) and end-systole (d)

with increasing exercise intensity $(119 \pm 10 \mathrm{mmHg}$ at rest to $143 \pm 15 \mathrm{mmHg}$ at low and $160 \pm 24 \mathrm{mmHg}$ at moderate exercise), whilst diastolic BP remained unchanged
Table 2 Comparisons of resting stroke volumes indexed to BSA between clinical and C-SENSE accelerated aortic and pulmonary flow sequences

\begin{tabular}{|c|c|c|c|c|c|}
\hline & \multirow[t]{3}{*}{$\mathrm{SV}\left(\mathrm{ml} / \mathrm{m}^{2}\right)$} & \multicolumn{4}{|c|}{ Flow comparison } \\
\hline & & \multicolumn{2}{|c|}{ Vs Clinical BH } & \multicolumn{2}{|l|}{ Vs Clinical FB } \\
\hline & & $\operatorname{Bias}\left(\mathrm{ml} / \mathrm{m}^{2}\right)$ & $\mathrm{CC}(\mathrm{r})$ & $\operatorname{Bias}\left(\mathrm{ml} / \mathrm{m}^{2}\right)$ & $\mathrm{CC}(\mathrm{r})$ \\
\hline \multicolumn{6}{|c|}{ Aortic flow } \\
\hline $\mathrm{BH}$ & $48.3 \pm 7.1$ & - & - & 0.11 & 0.762 \\
\hline FB & $48.4 \pm 5.7$ & -0.11 & 0.762 & - & - \\
\hline $\mathrm{CS} 3$ & $48.2 \pm 6.7$ & -0.12 & 0.96 & -0.23 & 0.865 \\
\hline CS6 & $46.2 \pm 6.4$ & -2.15 & 0.849 & -2.26 & 0.873 \\
\hline \multicolumn{6}{|c|}{ Pulmonary flow } \\
\hline $\mathrm{BH}$ & $48.9 \pm 5.9$ & - & - & 0.53 & 0.8 \\
\hline FB & $48.3 \pm 6.4$ & -0.53 & 0.8 & - & - \\
\hline $\mathrm{CS} 3$ & $48.2 \pm 7.6$ & -0.69 & 0.915 & -0.16 & 0.909 \\
\hline CS6 & $48.1 \pm 6.6$ & -0.73 & 0.85 & -0.20 & 0.655 \\
\hline
\end{tabular}

$B H$ breath hold, $C C$ correlation coefficient (Pearsons), CS3/CS6 compressed SENSE 3/6, $F B$ free-breathing, $S V$ stroke volume

$(71 \pm 8 \mathrm{mmHg}$ at rest to $76 \pm 13 \mathrm{mmHg}$ at low and $75 \pm 13 \mathrm{mmHg}$ at moderate exercise). BP was un-recordable at moderate exercise intensity in two subjects. Participants subjective rating of perceived exertion (RPE) on the Borg scale [23] were $9.6 \pm 1.8$ for low and $13.7 \pm 2.4$ for moderate exercise intensities, falling into the target ranges, as per ACSM guidelines [25], for the prescribed exercise 
Table 3 Image quality score comparison between flow sequences at rest and exercise

\begin{tabular}{|c|c|c|c|c|c|c|}
\hline \multirow[t]{2}{*}{ Flow sequence } & \multicolumn{2}{|l|}{ Resting } & \multicolumn{2}{|l|}{ Low exercise } & \multicolumn{2}{|c|}{ Moderate exercise } \\
\hline & Aortic & Pulmonary & Aortic & Pulmonary & Aortic & Pulmonary \\
\hline Clinical BH & $2.83 \pm 0.24^{*+\#}$ & $2.88 \pm 0.30 * \#$ & - & - & - & - \\
\hline Clinical FB & $2.21 \pm 0.38$ & $2.08 \pm 0.45^{\#}$ & - & - & - & - \\
\hline CS3 free-breathing & $2.33 \pm 0.3^{\#}$ & $2.38 \pm 0.58^{\#}$ & $1.5 \pm 0.41^{\#}$ & $1.46 \pm 0.62^{\#}$ & $1.21 \pm 0.25^{\#}$ & $1.08 \pm 0.19$ \\
\hline CS6 free-breathing & $1.75 \pm 0.32$ & $1.63 \pm 0.30$ & $1.33 \pm 0.37$ & $1.13 \pm 0.22$ & $0.88 \pm 0.46$ & $0.88 \pm 0.41$ \\
\hline
\end{tabular}

Image quality score: 3 - excellent, 2 - good, 1 - adequate $\& 0$ - non diagnostic

${ }^{*} \mathrm{p} \leq 0.05$ superior to clinical free-breathing sequence at same exercise stage, ${ }^{+} \mathrm{p} \leq 0.05$ superior to CS3 sequence at same exercise stage, ${ }^{\#} \mathrm{p} \leq 0.05$ superior to CS6 sequence at same exercise stage. $B H$ breath held, $C S$ compressed SENSE, $F B$ free-breathing
Fig. 4 Image quality of resting phase contrast image sequences, comparing both clinical standard sequences (Clinical breath held SENSE 2 \& clinical free-breathing no parallel imaging) with Compressed SENSE 3 and Compressed SENSE 6 sequences

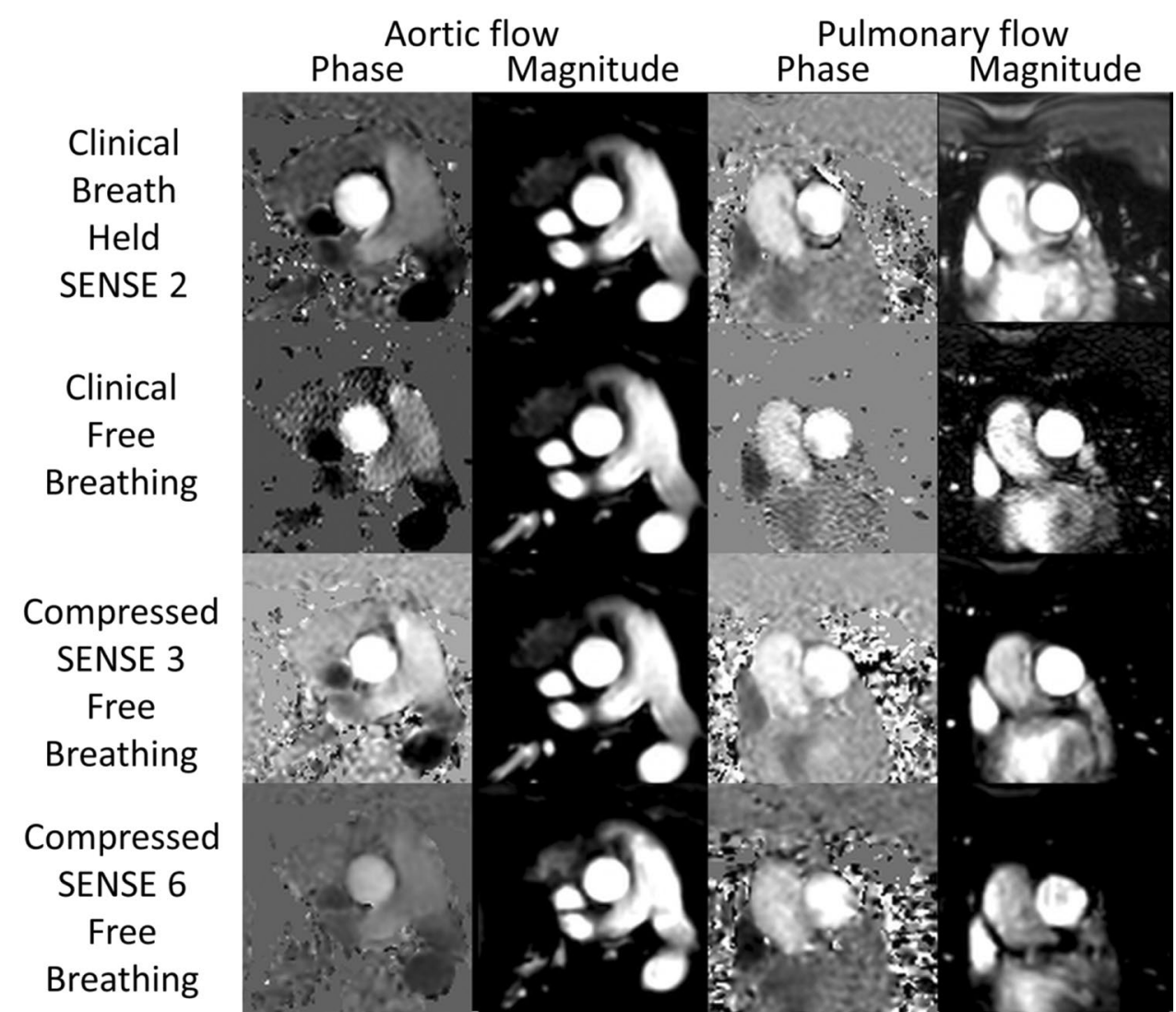

intensities. Therefore, both the objective haemodynamic and the subjective Borg RPE scores were within the advised ranges for the prescribed exercise intensities.

\section{Cardiac indices response to exercise}

\section{Volumes}

Table 4 demonstrates the cardiac volumetric and flow changes during exercise and Fig. 5 shows the typical image quality obtained during exercise for both cine and aortic and pulmonary PCMR images. During Ex-CMR, indexed left ventricular end-diastolic volume (LVEDVi) did not significantly alter $\left(88.5 \pm 16 \mathrm{ml} / \mathrm{m}^{2}\right.$ at rest, $88.2 \pm 15 \mathrm{ml} / \mathrm{m}^{2}$ at low an $85.9 \pm 14 \mathrm{ml} / \mathrm{m}^{2}$ at moderate, $\mathrm{p}=0.256$ for rest to moderate exercise), indexed LV stroke volume (LVSVi) increased significantly $\left(50 \pm 7 \mathrm{ml} / \mathrm{m}^{2}\right.$ at rest, $57.2 \pm 8 \mathrm{ml} / \mathrm{m}^{2}$ at low and $59.5 \pm 7 \mathrm{ml} / \mathrm{m}^{2}$ at moderate exercise; $\mathrm{p} \leq 0.001$ for rest to moderate exercise) driven by a significant fall in indexed LV end-systolic volume (LVESVi) $\left(38.4 \pm 11 \mathrm{ml} / \mathrm{m}^{2}\right.$ at rest vs $31 \pm 10 \mathrm{ml} / \mathrm{m}^{2}$ at low and $26.4 \pm 10 \mathrm{ml} / \mathrm{m}^{2}$ at $\mathrm{mod}-$ erate; $\mathrm{p} \leq 0.001$ for rest to moderate exercise) thus causing a rise in LV ejection fraction (LVEF) with exercise $(57 \pm 6 \%$ at rest, $66 \pm 7 \%$ at low and $70 \pm 8 \%$ at moderate exercise; $\mathrm{p} \leq 0.001$ for rest to moderate exercise). During Ex-CMR, right ventricular end-diastolic volume (RVEDVi) decreased 
Table 4 Haemodynamic response to supine bicycle exercise using the C-SENSE 3 protocol

\begin{tabular}{|c|c|c|c|c|c|c|c|}
\hline & Rest & Low & Moderate & ANOVA P-value & Rest vs Low & Low vs Mod & Rest vs Mod \\
\hline $\mathrm{HRR} \%$ Of $\mathrm{HR}_{\max }$ & N/A & $30-39 \%$ & $40-59 \%$ & - & - & - & - \\
\hline HR achieved & $58 \pm 6$ & $102 \pm 5$ & $119 \pm 5$ & $<0.001$ & $<0.001$ & $<0.001$ & $<0.001$ \\
\hline Systolic BP* & $119 \pm 10$ & $143 \pm 15$ & $160 \pm 24$ & $<0.001$ & 0.001 & 0.038 & $<0.001$ \\
\hline Diastolic BP* & $71 \pm 8$ & $76 \pm 13$ & $75 \pm 13$ & 0.605 & 1 & 1 & 1 \\
\hline Borg RPE & $6 \pm 0$ & $9.6 \pm 1.8$ & $13.7 \pm 2.4$ & $<0.001$ & $<0.001$ & $<0.001$ & $<0.001$ \\
\hline Cycle resistance $(\mathrm{W})$ & 0 & $52 \pm 26$ & $84 \pm 24$ & $<0.001$ & $<0.001$ & $<0.001$ & $<0.001$ \\
\hline LVEDV (ml) & $164 \pm 39$ & $163 \pm 36$ & $159 \pm 34$ & 0.052 & 1 & 0.187 & 0.192 \\
\hline LVEDVi (ml) & $88.5 \pm 16$ & $88.2 \pm 15$ & $85.9 \pm 14$ & 0.066 & 1 & 0.173 & 0.256 \\
\hline LVESV (ml) & $71 \pm 23$ & $58 \pm 21$ & $49 \pm 20$ & $<0.001$ & 0.001 & 0.001 & $<0.001$ \\
\hline LVESVi (ml) & $38.4 \pm 11$ & $31 \pm 10$ & $26.4 \pm 10$ & $<0.001$ & 0.001 & 0.001 & $<0.001$ \\
\hline LVSV (ml) & $93 \pm 19$ & $106 \pm 19$ & $110 \pm 19$ & $<0.001$ & 0.002 & 0.193 & $<0.001$ \\
\hline LVSVi (ml) & $50 \pm 7$ & $57.2 \pm 8$ & $59.5 \pm 7$ & $<0.001$ & 0.002 & 0.177 & $<0.001$ \\
\hline $\operatorname{LVEF}(\%)$ & $57 \pm 6$ & $66 \pm 7$ & $70 \pm 8$ & $<0.001$ & $<0.001$ & 0.002 & $<0.001$ \\
\hline Aortic SV (ml) & $89 \pm 17$ & $102 \pm 18$ & $105 \pm 18$ & $<0.001$ & 0.001 & 0.708 & $<0.001$ \\
\hline Aortic SVi (ml) & $48.2 \pm 7$ & $55.1 \pm 8$ & $56.6 \pm 8$ & $<0.002$ & 0.001 & 0.682 & $<0.001$ \\
\hline RVEDV (ml) & $166 \pm 34$ & $161 \pm 33$ & $158 \pm 31$ & 0.003 & 0.104 & 0.18 & 0.025 \\
\hline RVEDVi (ml) & $89.8 \pm 15$ & $87.2 \pm 15$ & $85.2 \pm 14$ & 0.002 & 0.096 & 0.16 & 0.023 \\
\hline RVESV (ml) & $75 \pm 21$ & $58 \pm 20$ & $48 \pm 17$ & $<0.001$ & $<0.001$ & 0.001 & $<0.001$ \\
\hline RVESVi (ml) & $40.4 \pm 10$ & $31.1 \pm 10$ & $25.8 \pm 8$ & $<0.001$ & $<0.001$ & 0.001 & $<0.001$ \\
\hline RVSV (ml) & $91 \pm 17$ & $104 \pm 18$ & $110 \pm 17$ & $<0.001$ & $<0.001$ & 0.008 & $<0.001$ \\
\hline RVSVi (ml) & $49.4 \pm 7$ & $56.1 \pm 7$ & $59.4 \pm 7$ & $<0.001$ & $<0.001$ & 0.008 & $<0.001$ \\
\hline RVEF (\%) & $56 \pm 6$ & $65 \pm 7$ & $70 \pm 6$ & $<0.001$ & $<0.001$ & $<0.001$ & $<0.001$ \\
\hline Pulmonary SV (ml) & $89 \pm 18$ & $100 \pm 17$ & $102 \pm 16$ & $<0.001$ & 0.007 & 1 & 0.012 \\
\hline Pulmonary SVi (ml) & $48.2 \pm 8$ & $54.3 \pm 7$ & $55.2 \pm 7$ & $<0.001$ & 0.005 & 1 & 0.009 \\
\hline
\end{tabular}

$B P$ blood pressure, $E D V$ end-diastolic volume, $E F$ ejection fraction, $E S V$ end-systolic volume, $H R$ heart rate, $i$ indexed to body surface area, $L V$ left ventricle, $R P E$ rate of perceived exertion, $R V$ right ventricle, $S V$ stroke volume

*Blood pressure was unrecordable in 2 patients at moderate exercise intensity

significantly $\left(89.8 \pm 15 \mathrm{ml} / \mathrm{m}^{2}\right.$ at rest, $87.2 \pm 15 \mathrm{ml} / \mathrm{m}^{2}$ at low and $85.2 \pm 14 \mathrm{ml} / \mathrm{m}^{2}$ at moderate exercise, $\mathrm{p}=0.023$ rest to moderate exercise), indexed right ventricular endsystolic volume (RVESVi) decreased $\left(40.4 \pm 10 \mathrm{ml} / \mathrm{m}^{2}\right.$ at rest vs $31.1 \pm 10 \mathrm{ml} / \mathrm{m}^{2}$ at low and $25.8 \pm 8 \mathrm{ml} / \mathrm{m}^{2}$ at moderate exercise; $\mathrm{p} \leq 0.001$ for rest to moderate exercise) driving a rise in indexed right ventricular stroke volume (RVSVi) $\left(49.4 \pm 7 \mathrm{ml} / \mathrm{m}^{2}\right.$ at rest, $56.1 \pm 7 \mathrm{ml} / \mathrm{m}^{2}$ at low and $59.4 \pm 7 \mathrm{ml} / \mathrm{m}^{2}$ at moderate; $\mathrm{p} \leq 0.001$ for rest to moderate exercise) and right ventricular ejection fraction (RVEF) $(56 \pm 6 \%$ vs $65 \pm 7 \%$ at low and $70 \pm 6 \%$ at moderate exercise; $p=<0.001$ for rest to moderate exercise) with increasing exercise.

\section{Flow}

Aortic stroke volumes increased significantly during Ex-CMR from $48.2 \pm 7 \mathrm{ml} / \mathrm{m}^{2} /$ cardiac cycle at rest to $55.1 \pm 8 \mathrm{ml} / \mathrm{m}^{2} /$ cardiac cycle at low and $56.6 \pm 8 \mathrm{ml} / \mathrm{m}^{2} /$ cardiac cycle at moderate exercise intensities $(\mathrm{p} \leq 0.001$, rest to moderate exercise). Aortic stroke volumes showed very strong correlation with LVSVi at rest $(r=0.93)$, low $(\mathrm{r}=0.97)$ and moderate exercise $(\mathrm{r}=0.98)$. During Ex-CMR pulmonary stroke volumes increased significantly from $48.2 \pm 8 \mathrm{ml} / \mathrm{m}^{2} /$ cardiac cycle at rest to $54.3 \pm 7 \mathrm{ml} / \mathrm{m}^{2} /$ cardiac cycle at low and $55.2 \pm 7 \mathrm{ml} / \mathrm{m}^{2} /$ cardiac cycle at moderate exercise intensities ( $\mathrm{p}=0.009$, rest to moderate exercise) and correlated strongly with RVSVi at rest $(r=0.88)$ and very strongly during low $(\mathrm{r}=0.90)$ and moderate exercise $(\mathrm{r}=0.97)$.

\section{Intra/inter-observer reproducibility}

Intra- and inter-observer reproducibility is shown in Table 5. Intra-observer reproducibility of all cardiac sequences assessed at rest and during exercise by $\mathrm{CV}$ were excellent $(\mathrm{CV}<10 \%)$ and all sequences assessed by ICC were excellent (ICC > 0.9) with exception of pulmonary flow at low $(\mathrm{ICC}=0.892)$ and moderate exercise $(\mathrm{ICC}=0.847)$ and LVSV at moderate exercise $(\mathrm{ICC}=0.897)$.

Inter-observer reproducibility assessed by $\mathrm{CV}$ of cardiac parameters were similarly excellent $(\mathrm{CV}<10 \%)$, with the 
Fig. 5 Typical image quality of cine and phase contrast imaging at rest and during Ex-CMR to low and moderate exercise

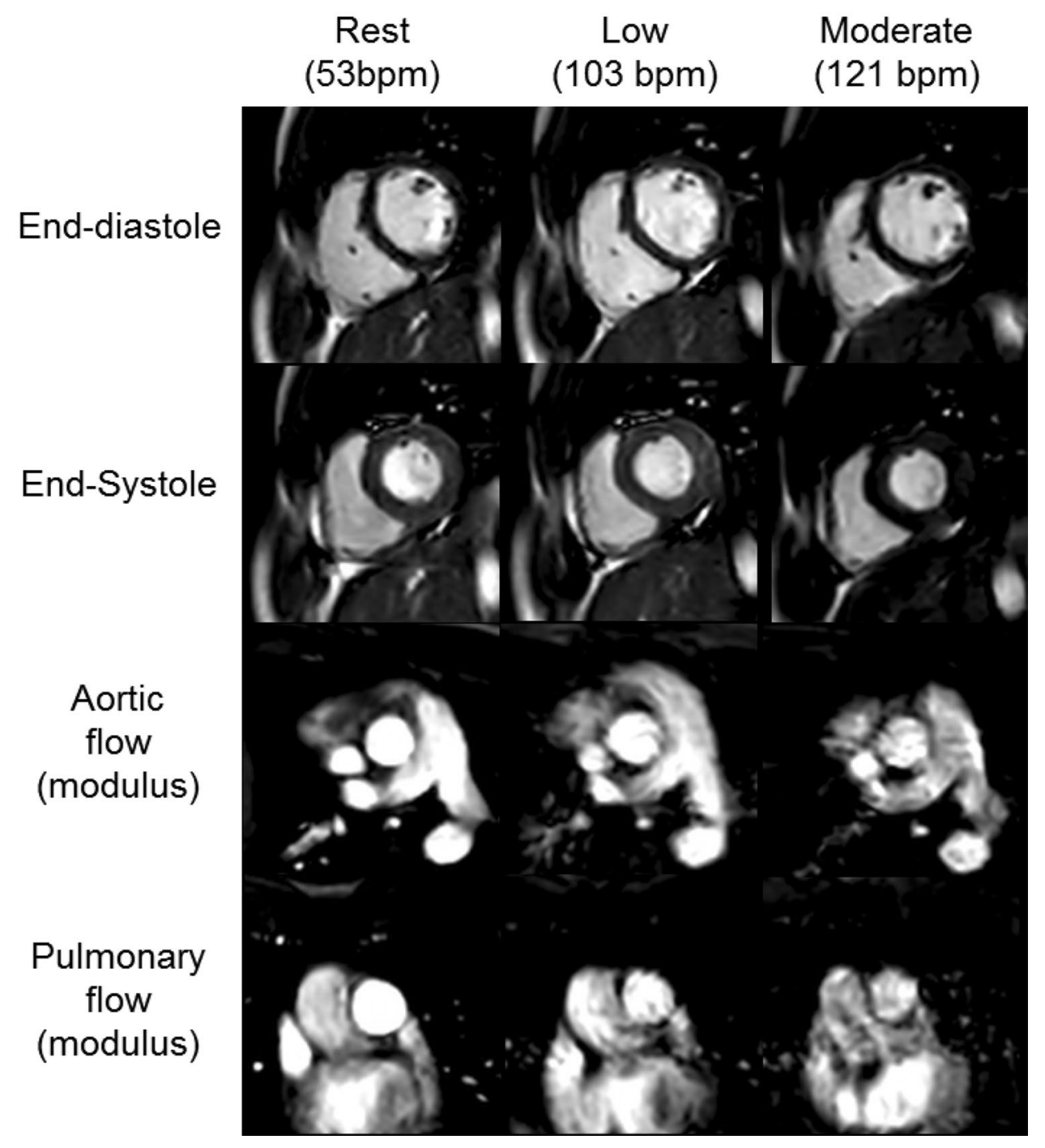

exception of RVESV by CS3 cine imaging at rest (CV 12.96\%) and LVESV and RVESV during exercise, with a CV of $11.38 \%$ and $11.39 \%$ at low and $16.61 \%$ and $17.93 \%$ at moderate exercise intensities respectively. Cardiac parameters demonstrated excellent ICC $(>0.9)$ at rest with the exception of RVSV \& RVEF on clinical sequences and RVESV, RVSV \& RVEF on CS3 sequences demonstrating good ICC (>0.8). During low intensity exercise all cardiac parameters demonstrated excellent ICC (>0.9), which decreased to good ICC at moderate exercise (ICC $>0.75$ ) with the exception of LVEDV and aortic flow which maintained excellent ICC $(>0.9)$. The increase in variability of end-systolic volumes with increased exercise intensity is not unsurprising given the significant fall in ESV with exercise which allows for a smaller margin of error.

\section{Discussion}

This study has shown that (1) it is feasible to assess biventricular volumes and flow by CMR during continuous in-scanner supine bicycle exercise using free-breathing C-SENSE, (2) Using CS3 compared to standard clinical imaging, image quality and reproducibility were good, but this was not the case with higher acceleration factors (CS6) and (3) Using CS3, we have shown superior reproducibility in comparison to the only previous study to perform biventricular volume and flow assessment during continuous ExCMR (which used un-gated real-time sequences) [18].

To our knowledge [20], only one prior study, by Jaijee et al., has assessed biventricular volume and flow assessment with free-breathing during continuous exercise, and did so by utilising an un-gated real-time technique [18]. The study was insightful, investigating right ventricular dysfunction in acute hypoxia and chronic pulmonary arterial hypertension. However the authors didn't perform image quality 
Table 5 Coefficient of Variation and intra-class correlation coefficient for the reproducibility of biventricular volumetric and flow indices

\begin{tabular}{|c|c|c|c|c|c|}
\hline \multirow[t]{2}{*}{ Exercise level and sequence } & \multirow[t]{2}{*}{ Cardiac parameter } & \multicolumn{2}{|c|}{ Intra-observer } & \multicolumn{2}{|c|}{ Inter-observer } \\
\hline & & $\mathrm{COV}$ & ICC & $\mathrm{COV}$ & $\mathrm{ICC}$ \\
\hline \multirow[t]{12}{*}{ Resting clinical } & LVEDV & 1.32 & 0.996 & 2.12 & 0.988 \\
\hline & LVESV & 2.69 & 0.989 & 6.58 & 0.968 \\
\hline & LVSV & 2.47 & 0.967 & 4.24 & 0.920 \\
\hline & LVEF & 2.06 & 0.987 & 3.75 & 0.931 \\
\hline & RVEDV & 2.29 & 0.985 & 2.65 & 0.979 \\
\hline & RVESV & 5.34 & 0.968 & 8.60 & 0.918 \\
\hline & RVSV & 3.94 & 0.953 & 6.89 & 0.808 \\
\hline & RVEF & 3.55 & 0.957 & 6.35 & 0.877 \\
\hline & Aortic flow FB & 1.14 & 0.990 & 3.07 & 0.930 \\
\hline & Aortic flow BH & 0.83 & 0.997 & 2.05 & 0.980 \\
\hline & Pulmonary flow FB & 1.18 & 0.993 & 2.15 & 0.973 \\
\hline & Pulmonary flow BH & 1.40 & 0.988 & 1.78 & 0.981 \\
\hline \multirow[t]{10}{*}{ Resting compressed SENSE 3 free-breathing } & LVEDV & 1.29 & 0.995 & 2.50 & 0.985 \\
\hline & LVESV & 3.89 & 0.976 & 6.66 & 0.965 \\
\hline & LVSV & 2.89 & 0.958 & 3.44 & 0.942 \\
\hline & LVEF & 2.98 & 0.974 & 3.41 & 0.953 \\
\hline & RVEDV & 1.92 & 0.986 & 4.15 & 0.937 \\
\hline & RVESV & 5.40 & 0.957 & 12.96 & 0.814 \\
\hline & RVSV & 3.67 & 0.945 & 6.24 & 0.824 \\
\hline & RVEF & 3.23 & 0.968 & 7.26 & 0.817 \\
\hline & Aortic flow & 0.83 & 1.000 & 1.19 & 0.993 \\
\hline & Pulmonary flow & 2.19 & 0.986 & 3.55 & 0.950 \\
\hline \multirow[t]{10}{*}{ Low intensity exercise (CS3 free-breathing) } & LVEDV & 0.76 & 0.998 & 3.97 & 0.953 \\
\hline & LVESV & 8.77 & 0.915 & 11.38 & 0.911 \\
\hline & LVSV & 4.44 & 0.907 & 3.46 & 0.952 \\
\hline & LVEF & 4.72 & 0.923 & 4.08 & 0.916 \\
\hline & RVEDV & 1.95 & 0.984 & 3.72 & 0.955 \\
\hline & RVESV & 8.78 & 0.907 & 11.39 & 0.909 \\
\hline & RVSV & 2.97 & 0.947 & 3.07 & 0.940 \\
\hline & RVEF & 4.37 & 0.934 & 4.09 & 0.908 \\
\hline & Aortic flow & 1.99 & 0.986 & 5.88 & 0.917 \\
\hline & Pulmonary flow & 3.13 & 0.892 & 3.84 & 0.927 \\
\hline \multirow[t]{10}{*}{ Moderate intensity exercise (CS3 free-breathing) } & LVEDV & 2.09 & 0.986 & 4.27 & 0.940 \\
\hline & LVESV & 9.50 & 0.952 & 16.61 & 0.883 \\
\hline & LVSV & 4.17 & 0.897 & 4.37 & 0.849 \\
\hline & LVEF & 3.54 & 0.956 & 4.96 & 0.891 \\
\hline & RVEDV & 3.48 & 0.964 & 5.77 & 0.878 \\
\hline & RVESV & 9.23 & 0.926 & 17.93 & 0.754 \\
\hline & RVSV & 3.77 & 0.923 & 4.99 & 0.830 \\
\hline & RVEF & 2.95 & 0.955 & 5.12 & 0.837 \\
\hline & Aortic flow & 2.22 & 0.975 & 4.01 & 0.918 \\
\hline & Pulmonary flow & 6.22 & 0.847 & 6.11 & 0.879 \\
\hline
\end{tabular}

$C O V$ co-efficient of variance, $E D V$ end-diastolic volume, $E F$ ejection fraction, $E S V$ end-systolic volume, $H R$ heart rate, $i$ indexed to body surface area, $I C C$ intra-class correlation, $L V$ left ventricle, $R V$ right ventricle assessment and demonstrated suboptimal reproducibility, on the basis of ICC for intra- and inter-observer variability for RVEF. Our RVEF ICC for intra- and inter-observer analysis respectively was 0.968 and 0.817 at rest, and 0.955 and 0.837 at moderate exercise (vs 0.71 and 0.85 at rest and 0.625 and 0.744 at moderate exercise in the un-gated 
real-time study). One caveat with this direct comparison is we only studied healthy volunteers, whereas Jaijee et al. studied healthy volunteers and patients with pulmonary hypertension [18]; patients may demonstrate increased respiratory motion, worse image quality and so a resultant decrease in reproducibility. Therefore our technique needs testing in patients with cardiac disease before direct comparisons can be confidently made. Both studies represent a significant progression in the potential clinical utility of Ex-CMR, however our study is the first study to demonstrate such feasibility using vendor provided sequences with analysis performed on standard commercially available software.

Comparatively lower heart rates are observed during supine exercise compared with upright exercise at the same intensity. Exercise in the supine position results in higher blood pressure than upright exercise [26], therefore a similar double product (systolic blood pressure $\mathrm{x}$ heart rate), which is an index of myocardial oxygen consumption [27], is achieved at lower heart rates than upright exercise [28-30]. Therefore, we used heart rate reserve (HRR) to determine subject specific target heart rates, with the resting heart rate assessed when supine. Importantly, our study aimed only to assess subjects to moderate exercise intensity, and not to submaximal or maximal intensity. Maximal in-scanner continuous exercise can create significant motion artefacts, rendering images non-diagnostic, but more importantly may be unsafe in a patient population, given the inability to accurately assess ST segment changes which could prompt test termination. However, even at moderate intensity exercise, an Ex-CMR protocol assessing biventricular function and flow, may theoretically provide additional diagnostic and prognostic information in valvular and congenital heart disease, especially for valvular regurgitation assessment.

The haemodynamic response to exercise demonstrated a minimal change in LVEDV and a rise in LVSV driven by a fall in LVESV during exercise, which is in keeping with a recent Ex-CMR meta-analysis of 16 Ex-CMR studies [31]. Indeed, our study demonstrated a non-significant decrease in LVEDV with exercise as was demonstrated by the majority of Ex-CMR studies in the Ex-CMR meta-analysis. These findings replicate the theory that being truly supine (rather than recumbent in stress echocardiography) results in near maximal LVEDV at rest and thus no significant increase is seen with exercise.

\section{Clinical implications}

The clinical utility of Ex-CMR requires rapid image acquisition using accessible free-breathing sequences and analysis software. We demonstrated this is feasible using C-SENSE. C-SENSE is vendor provided, boasting faster image acquisition [32, 33] and greater robustness to respiratory motion [19] than standard parallel imaging techniques. Our
C-SENSE protocol's ability to assess biventricular haemodynamics and great vessel flow, which could be used to quantify valvular flow/regurgitant flow, in response to incremental exercise could theoretically provide a comprehensive assessment in valvular and congenital heart disease. Further research in these patient cohorts is required. In asymptomatic significant valve disease, ventricular dilatation/dysfunction or an abnormal exercise response can guide the decision to advise intervention [1,34]. Given CMR is the reference standard for biventricular assessment and CMR derived aortic and mitral regurgitation quantification boasts superior prognostic value to transthoracic echocardiography [35-37], the additional assessment during exercise may hypothetically provide further prognostic information. Additionally, in-scanner MR-CPET is feasible [38] and our protocol could be performed in combination, theoretically creating a single comprehensive investigation. C-SENSE acceleration may benefit other Ex-CMR applications. For example, free breathing first pass perfusion using compressed sensing at rest [39] and supine exercise stress perfusion CMR are both feasible [40], therefore C-SENSE accelerated Ex-CMR stress perfusion may also be feasible. Our technique requires further research to demonstrate feasibility in patient populations, assess if additional prognostic information is provided above a resting CMR scan and whether C-SENSE can be used for other Ex-CMR applications.

\section{Study limitations}

The study sample size is small and in healthy volunteers with a healthy mean BMI $\left(23.9 \pm 2.3 \mathrm{~kg} / \mathrm{m}^{2}\right)$ and a mean age ( $35 \pm 9$ years) younger than patients typically referred for exercise cardiac imaging. Supine Ex-CMR is feasible in older patients [41-44] and obese patients [45] but may be tolerated less well than by our study population, potentially resulting in more respiratory and motion artefacts. Thus our technique requires further evaluation in patients with cardiovascular disease. Derived volumes and flow from biventricular cine images and phase contrast images respectively were not compared directly with the reference standard of the direct Fick method, however as we have demonstrated, the biventricular cine and corresponding phase contrast flow stroke volumes correlated very strongly, demonstrating the internal validity of our technique. Additionally, our results follow prior supine Ex-CMR studies, as demonstrated in a recent meta-analysis [31], demonstrating rising stroke volumes with increasing exercise driven by a fall in LVESV but minimal change in LVEDV. Inter-scan reproducibility was not assessed with this study, but has been demonstrated in our institution previously in an Ex-CMR study assessing biventricular volumes using a similar retrospectively gated, respiratory navigated short axis cine sequence [42]. As expected, and demonstrated in prior Ex-CMR studies 
$[16,31,46,47]$, image quality decreases with increasing exercise intensity, however our study still demonstrated good intra- and inter-observer reproducibility during moderate intensity exercise. ECG interference was encountered in one patient, early in the study, such that miss-triggering occurred at moderate exercise intensity. This made analysis technically unfeasible and so the subject was excluded from the study. Subsequent subjects had pulse oximetry attached as a backup cardiac gating technique should ECG interference occur, however this was not required.

\section{Conclusion}

Assessment of biventricular function, aortic and pulmonary flows during continuous exercise is feasible during exercise to moderate intensity using a free-breathing C-SENSE accelerated protocol. The ability to use commercially available analysis software with this vendor provided technique increases the potential clinical utility of Ex-CMR. The developed protocol allows the direct quantification of flow across the aortic and pulmonary valves and indirect quantification of mitral and tricuspid regurgitation during exercise. Further evaluation is needed in patients with cardiovascular disease to assess the value and reproducibility in a clinical setting.

\section{Compliance with ethical standards}

Conflict of interest Dr David M. Higgins is an employee of Phillips, but did not receive specific funding for this study.

Open Access This article is licensed under a Creative Commons Attribution 4.0 International License, which permits use, sharing, adaptation, distribution and reproduction in any medium or format, as long as you give appropriate credit to the original author(s) and the source, provide a link to the Creative Commons licence, and indicate if changes were made. The images or other third party material in this article are included in the article's Creative Commons licence, unless indicated otherwise in a credit line to the material. If material is not included in the article's Creative Commons licence and your intended use is not permitted by statutory regulation or exceeds the permitted use, you will need to obtain permission directly from the copyright holder. To view a copy of this licence, visit http://creativecommons.org/licenses/by/4.0/.

\section{References}

1. Baumgartner H, Falk V, Bax JJ, De Bonis M, Hamm C, Holm PJ, Iung B, Lancellotti P, Lansac E, Rodriguez Munoz D, Rosenhek R, Sjogren J, Tornos Mas P, Vahanian A, Walther T, Wendler O, Windecker S, Zamorano JL (2017) 2017 ESC/EACTS Guidelines for the management of valvular heart disease. Eur Heart J 38(36):2739-2791. https://doi.org/10.1093/eurheartj/ehx391
2. Stout KK, Daniels CJ, Aboulhosn JA, Bozkurt B, Broberg CS, Colman JM, Crumb SR, Dearani JA, Fuller S, Gurvitz M, Khairy P, Landzberg MJ, Saidi A, Valente AM, Van Hare GF (2019) 2018 AHA/ACC guideline for the management of adults with congenital heart disease: executive summary: a report of the american college of cardiology/american heart association task force on clinical practice guidelines. J Am Coll Cardiol 73(12):1494-1563. https://doi.org/10.1016/j.jacc.2018.08.1028

3. Bokhari S, Shahzad A, Bergmann SR (2008) Superiority of exercise myocardial perfusion imaging compared with the exercise ECG in the diagnosis of coronary artery disease. Coron Artery Dis 19(6):399-404. https://doi.org/10.1097/MCA.0b013e3283 $021 \mathrm{ab} 4$

4. Jeetley P, Burden L, Senior R (2006) Stress echocardiography is superior to exercise ECG in the risk stratification of patients presenting with acute chest pain with negative Troponin. Eur J Echocardiogr 7(2):155-164. https://doi.org/10.1016/j. euje.2005.05.002

5. Bellenger NG, Burgess MI, Ray SG, Lahiri A, Coats AJ, Cleland JG, Pennell DJ (2000) Comparison of left ventricular ejection fraction and volumes in heart failure by echocardiography, radionuclide ventriculography and cardiovascular magnetic resonance; are they interchangeable? Eur Heart J 21(16):1387-1396. https:// doi.org/10.1053/euhj.2000.2011

6. Greenwood JP, Maredia N, Younger JF, Brown JM, Nixon J, Everett CC, Bijsterveld P, Ridgway JP, Radjenovic A, Dickinson CJ, Ball SG, Plein S (2012) Cardiovascular magnetic resonance and single-photon emission computed tomography for diagnosis of coronary heart disease (CE-MARC): a prospective trial. Lancet 379(9814):453-460. https://doi.org/10.1016/S0140 $-6736(11) 61335-4$

7. Greenwood JP, Maredia N, Radjenovic A, Brown JM, Nixon J, Farrin AJ, Dickinson C, Younger JF, Ridgway JP, Sculpher M, Ball SG, Plein S (2009) Clinical evaluation of magnetic resonance imaging in coronary heart disease: the CE-MARC study. Trials 10:62. https://doi.org/10.1186/1745-6215-10-62

8. Greenwood JP, Herzog BA, Brown JM, Everett CC, Nixon J, Bijsterveld P, Maredia N, Motwani M, Dickinson CJ, Ball SG, Plein S (2016) Prognostic Value of Cardiovascular Magnetic Resonance and Single-Photon Emission Computed Tomography in Suspected Coronary Heart Disease: Long-Term Follow-up of a Prospective, Diagnostic Accuracy Cohort Study. Ann Internal Med. https://doi. org/10.7326/M15-1801

9. Lancellotti P, Pellikka PA, Budts W, Chaudhry FA, Donal E, Dulgheru R, Edvardsen T, Garbi M, Ha JW, Kane GC, Kreeger J, Mertens L, Pibarot P, Picano E, Ryan T, Tsutsui JM, Varga A (2017) The clinical use of stress echocardiography in non-ischaemic heart disease: recommendations from the European Association of Cardiovascular Imaging and the American Society of Echocardiography. J Am Soc Echocardiogr 30(2):101-138. https ://doi.org/10.1016/j.echo.2016.10.016

10. Varga A, Garcia MA, Picano E, International Stress Echo Complication R (2006) Safety of stress echocardiography (from the International Stress Echo Complication Registry). Am J Cardiol 98(4):541-543. https://doi.org/10.1016/j.amjcard.2006.02.064

11. Pellikka PA, Nagueh SF, Elhendy AA, Kuehl CA, Sawada SG, American Society of E (2007) American Society of Echocardiography recommendations for performance, interpretation, and application of stress echocardiography. J Am Soc Echocardiogr 20(9):1021-1041. https://doi.org/10.1016/j.echo.2007.07.00310 $.1016 /$ j.echo.2007.07.003

12. Wolk MJ, Bailey SR, Doherty JU, Douglas PS, Hendel RC, Kramer CM, Min JK, Patel MR, Rosenbaum L, Shaw LJ, Stainback RF, Allen JM (2014) ACCF/AHA/ASE/ASNC/HFSA/HRS/ SCAI/SCCT/SCMR/STS 2013 multimodality appropriate use criteria for the detection and risk assessment of stable ischemic 
heart disease: a report of the American College of Cardiology Foundation Appropriate Use Criteria Task Force, American Heart Association, American Society of Echocardiography, American Society of Nuclear Cardiology, Heart Failure Society of America, Heart Rhythm Society, Society for Cardiovascular Angiography and Interventions, Society of Cardiovascular Computed Tomography, Society for Cardiovascular Magnetic Resonance, and Society of Thoracic Surgeons. J Am Coll Cardiol 63(4):380-406. https:// doi.org/10.1016/j.jacc.2013.11.009

13. Raman SV, Dickerson JA, Mazur W, Wong TC, Schelbert EB, Min JK, Scandling D, Bartone C, Craft JT, Thavendiranathan P, Mazzaferri EL Jr, Arnold JW, Gilkeson R, Simonetti OP (2016) Diagnostic performance of treadmill exercise cardiac magnetic resonance: the prospective, multicenter exercise CMR's accuracy for cardiovascular stress testing (EXACT) trial. J Am Heart Assoc. https://doi.org/10.1161/JAHA.116.003811

14. La Gerche A, Claessen G, Van de Bruaene A, Pattyn N, Van Cleemput J, Gewillig M, Bogaert J, Dymarkowski S, Claus P, Heidbuchel H (2013) Cardiac MRI: a new gold standard for ventricular volume quantification during high-intensity exercise. Circ Cardiovasc Imaging 6(2):329-338. https://doi.org/10.1161/CIRCI MAGING.112.980037

15. Roest AA, Kunz P, Lamb HJ, Helbing WA, van der Wall EE, de Roos A (2001) Biventricular response to supine physical exercise in young adults assessed with ultrafast magnetic resonance imaging. Am J Cardiol 87(5):601-605

16. Lurz P, Muthurangu V, Schievano S, Nordmeyer J, Bonhoeffer P, Taylor AM, Hansen MS (2009) Feasibility and reproducibility of biventricular volumetric assessment of cardiac function during exercise using real-time radial k-t SENSE magnetic resonance imaging. J Magnet Reson Imaging 29(5):1062-1070. https://doi. org/10.1002/jmri.21762

17. Barber NJ, Ako EO, Kowalik GT, Cheang MH, Pandya B, Steeden JA, Moledina S, Muthurangu V (2016) Magnetic resonanceaugmented cardiopulmonary exercise testing: comprehensively assessing exercise intolerance in children with cardiovascular disease. Circ Cardiovas Imaging. https://doi.org/10.1161/CIRCI MAGING.116.005282

18. Jaijee S, Quinlan M, Tokarczuk P, Clemence M, Howard L, Gibbs JSR, O'Regan DP (2018) Exercise cardiac MRI unmasks right ventricular dysfunction in acute hypoxia and chronic pulmonary arterial hypertension. Am J Physiol Heart Circ Physiol 315(4):H950-H957. https://doi.org/10.1152/ajpheart.00146.2018

19. Kido T, Nakamura M, Watanabe K, Schmidt M, Forman C, Mochizuki T (2017) Assessment of left ventricular function and mass on free-breathing compressed sensing real-time cine imaging. Circ J 81(10):1463-1468. https://doi.org/10.1253/circj .CJ-17-0123

20. Craven TP, Tsao CW, La Gerche A, Simonetti OP, Greenwood JP (2020) Exercise cardiovascular magnetic resonance: development, current utility and future applications. J Cardiovasc Magnet Reson 22(1):65. https://doi.org/10.1186/s12968-020-00652-w

21. Fletcher GF, Ades PA, Kligfield P, Arena R, Balady GJ, Bittner VA, Coke LA, Fleg JL, Forman DE, Gerber TC, Gulati M, Madan K, Rhodes J, Thompson PD, Williams MA, American Heart Association Exercise CR, Prevention Committee of the Council on Clinical Cardiology CoNPA, Metabolism CoC, Stroke N, Council on E, Prevention (2013) Exercise standards for testing and training: a scientific statement from the American Heart Association. Circulation 128(8):873-934. https://doi.org/10.1161/CIR.0b013 e31829b5b44

22. Tanaka H, Monahan KD, Seals DR (2001) Age-predicted maximal heart rate revisited. J Am Coll Cardiol 37(1):153-156
23. Borg G (1990) Psychophysical scaling with applications in physical work and the perception of exertion. Scand J Work Environ Health 16(1):55-58. https://doi.org/10.5271/sjweh.1815

24. Heiberg J, Asschenfeldt B, Maagaard M, Ringgaard S (2017) Dynamic bicycle exercise to assess cardiac output at multiple exercise levels during magnetic resonance imaging. Clin Imaging 46:102-107. https://doi.org/10.1016/j.clinimag.2017.07.010

25. Garber CE, Blissmer B, Deschenes MR, Franklin BA, Lamonte MJ, Lee IM, Nieman DC, Swain DP (2011) American College of Sports Medicine position stand: Quantity and quality of exercise for developing and maintaining cardiorespiratory, musculoskeletal, and neuromotor fitness in apparently healthy adults: guidance for prescribing exercise. Med Sci Sports Exercise 43(7):13341359. https://doi.org/10.1249/MSS.0b013e318213fefb

26. Quinn TJ, Smith SW, Vroman NB, Kertzer R, Olney WB (1995) Physiologic responses of cardiac patients to supine, recumbent, and upright cycle ergometry. Arch Phys Med Rehabil 76(3):257-261

27. Nelson RR, Gobel FL, Jorgensen CR, Wang K, Wang Y, Taylor HL (1974) Hemodynamic predictors of myocardial oxygen consumption during static and dynamic exercise. Circulation 50(6):1179-1189

28. Badruddin SM, Ahmad A, Mickelson J, Abukhalil J, Winters WL, Nagueh SF, Zoghbi WA (1999) Supine bicycle versus post-treadmill exercise echocardiography in the detection of myocardial ischemia: a randomized single-blind crossover trial. J Am Coll Cardiol 33(6): 1485-1490

29. Peteiro J, Bouzas-Mosquera A, Estevez R, Pazos P, Pineiro M, Castro-Beiras A (2012) Head-to-head comparison of peak supine bicycle exercise echocardiography and treadmill exercise echocardiography at peak and at post-exercise for the detection of coronary artery disease. J Am Soc Echocardiogr 25(3):319-326. https ://doi.org/10.1016/j.echo.2011.11.002

30. Wicks JR, Sutton JR, Oldridge NB, Jones NL (1978) Comparison of the electrocardiographic changes induced by maximam exercise testing with treadmill and cycle ergometer. Circulation 57(6):1066-1070

31. Beaudry RI, Samuel TJ, Wang J, Tucker WJ, Haykowsky MJ, Nelson MD (2018) Exercise cardiac magnetic resonance imaging: a feasibility study and meta-analysis. Am J Physiol Regul Integr Comp Physiol 315(4):R638-R645. https://doi.org/10.1152/ajpre gu.00158.2018

32. Ma Y, Hou Y, Ma Q, Wang X, Sui S, Wang B (2019) Compressed SENSE single-breath-hold and free-breathing cine imaging for accelerated clinical evaluation of the left ventricle. Clin Radiol 74(4):e329-e325. https://doi.org/10.1016/j.crad.2018.12.012

33. Kocaoglu M, Pednekar AS, Wang H, Alsaied T, Taylor MD, Rat$\tan$ MS (2020) Breath-hold and free-breathing quantitative assessment of biventricular volume and function using compressed SENSE: a clinical validation in children and young adults. $\mathrm{J}$ Cardiovasc Magnetic Resonan 22(1):54. https://doi.org/10.1186/ s12968-020-00642-y

34. Nishimura RA, Otto CM, Bonow RO, Carabello BA, Erwin JP 3rd, Guyton RA, O'Gara PT, Ruiz CE, Skubas NJ, Sorajja P, Sundt TM 3rd, Thomas JD, Anderson JL, Halperin JL, Albert NM, Bozkurt B, Brindis RG, Creager MA, Curtis LH, DeMets D, Guyton RA, Hochman JS, Kovacs RJ, Ohman EM, Pressler SJ, Sellke FW, Shen WK, Stevenson WG, Yancy CW (2014) 2014 AHA/ACC guideline for the management of patients with valvular heart disease: a report of the American College of Cardiology/American Heart Association Task Force on Practice Guidelines. J Thorac Cardiovasc Surg 148(1):e1-e132. https:// doi.org/10.1016/j.jtcvs.2014.05.014

35. Myerson SG, d'Arcy J, Christiansen JP, Dobson LE, Mohiaddin R, Francis JM, Prendergast B, Greenwood JP, Karamitsos TD, 
Neubauer S (2016) Determination of clinical outcome in mitral regurgitation with cardiovascular magnetic resonance quantification. Circulation 133(23):2287-2296. https://doi.org/10.1161/ CIRCULATIONAHA.115.017888

36. Myerson SG, d'Arcy J, Mohiaddin R, Greenwood JP, Karamitsos TD, Francis JM, Banning AP, Christiansen JP, Neubauer S (2012) Aortic regurgitation quantification using cardiovascular magnetic resonance: association with clinical outcome. Circulation 126(12):1452-1460. https://doi.org/10.1161/CIRCULATIO NAHA.111.083600

37. Penicka M, Vecera J, Mirica DC, Kotrc M, Kockova R, Van Camp G (2018) Prognostic implications of magnetic resonance-derived quantification in asymptomatic patients with organic mitral regurgitation: comparison with doppler echocardiography-derived integrative approach. Circulation 137(13):1349-1360. https:// doi.org/10.1161/CIRCULATIONAHA.117.029332

38. Barber NJ, Ako EO, Kowalik GT, Steeden JA, Pandya B, Muthurangu V (2015) MR augmented cardiopulmonary exercise testing-a novel approach to assessing cardiovascular function. Physiol Meas 36(5):N85-94. https://doi.org/10.1088/0967-3334/36/5/N85

39. Otazo R, Kim D, Axel L, Sodickson DK (2010) Combination of compressed sensing and parallel imaging for highly accelerated first-pass cardiac perfusion MRI. Magn Reson Med 64(3):767776. https://doi.org/10.1002/mrm.22463

40. Pflugi S, Roujol S, Akcakaya M, Kawaji K, Foppa M, Heydari B, Goddu B, Kissinger K, Berg S, Manning WJ, Kozerke S, Nezafat R (2015) Accelerated cardiac MR stress perfusion with radial sampling after physical exercise with an MR-compatible supine bicycle ergometer. Magn Reson Med 74(2):384-395. https://doi. org $/ 10.1002 / \mathrm{mrm} .25405$

41. Roberts PA, Lin ACW, Cowan BR, Young AA, Stewart R (2018) Comparison of effects of losartan and metoprolol on left ventricular and aortic function at rest and during exercise in chronic aortic regurgitation. Int J Cardiovasc Imaging 34(4):615-624. https:// doi.org/10.1007/s10554-017-1268-y

42. Chew PG, Swoboda PP, Ferguson C, Garg P, Cook AL, Ibeggazene S, Brown LAE, Craven TP, Foley JR, Fent GJ, Saunderson
CE, Higgins DM, Plein S, Birch KM, Greenwood JP (2020) Feasibility and reproducibility of a cardiovascular magnetic resonance free-breathing, multi-shot, navigated image acquisition technique for ventricular volume quantification during continuous exercise. Quant Imaging Med Surg 10(9):1837-1851. https://doi. org/10.21037/qims-20-117

43. Nakamori S, Fahmy A, Jang J, El-Rewaidy H, Neisius U, Berg S, Goddu B, Pierce P, Rodriguez J, Hauser T, Ngo LH, Manning WJ, Nezafat R (2019) Changes in myocardial native T1 and T2 after exercise stress: a noncontrast CMR pilot study. JACC Cardiovasc Imaging. https://doi.org/10.1016/j.jcmg.2019.05.019

44. Schnell F, Claessen G, La Gerche A, Claus P, Bogaert J, Delcroix M, Carre F, Heidbuchel H (2017) Atrial volume and function during exercise in health and disease. J Cardiovasc Magnet Resonance 19(1):104. https://doi.org/10.1186/s12968-017-0416-9

45. Pinto TE, Gusso S, Hofman PL, Derraik JG, Hornung TS, Cutfield WS, Baldi JC (2014) Systolic and diastolic abnormalities reduce the cardiac response to exercise in adolescents with type 2 diabetes. Diabetes Care 37(5):1439-1446. https://doi.org/10.2337/ dc13-2031

46. Steding-Ehrenborg K, Jablonowski R, Arvidsson PM, Carlsson M, Saltin B, Arheden H (2013) Moderate intensity supine exercise causes decreased cardiac volumes and increased outer volume variations: a cardiovascular magnetic resonance study. J Cardiovasc Magn resonance 15:96. https://doi.org/10.1186/1532-429X-15-96

47. Asschenfeldt B, Heiberg J, Ringgaard S, Maagaard M, Redington A, Hjortdal VE (2017) Impaired cardiac output during exercise in adults operated for ventricular septal defect in childhood: a hitherto unrecognised pathophysiological response. Cardiol Young 27(8):1591-1598. https://doi.org/10.1017/S1047951117000877

Publisher's Note Springer Nature remains neutral with regard to jurisdictional claims in published maps and institutional affiliations. 